\title{
Antichiral ferromagnetism
}

\author{
Filipp N. Rybakov $\odot,{ }^{1, *}$ Anastasiia Pervishko, ${ }^{2}$ Olle Eriksson, ${ }^{3}$ and Egor Babaev ${ }^{1}$ \\ ${ }^{1}$ Department of Physics, KTH Royal Institute of Technology, SE-10691 Stockholm, Sweden \\ ${ }^{2}$ Skolkovo Institute of Science and Technology, Moscow 121205, Russia \\ ${ }^{3}$ Department of Physics and Astronomy, Uppsala University, Box 516, SE-75120 Uppsala, Sweden
}

(Received 22 December 2020; revised 18 March 2021; accepted 25 June 2021; published 13 July 2021)

\begin{abstract}
Here, by combining a symmetry-based analysis with numerical computations, we predict a different kind of magnetic ordering - antichiral ferromagnetism. This term aims to reflect that spontaneous modulation of the magnetization direction $\mathbf{m}(\mathbf{r})$ appears in a way that both types of chirality (handedness) exist simultaneously, and alternate in space. Without loss of generality, we focus our investigation on crystals with full tetrahedral symmetry where chiral interaction terms - Lifshitz invariants - are forbidden by symmetry. However, we demonstrate that the leading chirality-related term leads to nontrivial smooth magnetic textures exhibiting antichirality. In addition to the unconventional ground state, the revealed ordering gives rise to rich phenomena such as unique magnetic domains and skyrmions.
\end{abstract}

DOI: 10.1103/PhysRevB.104.L020406

Chirality (handedness) and chiral textures is a cornerstone concept in many fields of physics ranging from cosmology to nuclear and elementary particle physics [1]. In many materials, a chiral crystal structure results in a chiral ferromagnetic ordering $[2,3]$. The chiral textures in magnetism attracted renewed interest in recent decades owing to their potential relevance to technological applications, including alternative logic devices and racetrack memory where the information is encoded by virtue of magnetic textures [4-7]. Interestingly, some of the concepts developed so far are intended for the use of materials that have ferromagnetic ordering at the atomistic level, while at the mesoscale the direction of magnetization $\mathbf{m}(\mathbf{r})$ is modulated. The corresponding basic types of modulations are one dimensional and conventionally called cycloid [Fig. 1(a)], helix [Fig. 1(b)], spiral, screw, to name a few [8,9]. Such textures, as well as arbitrary vector fields of constant length, are characterized by geometrical invariants, the values of which at any point are independent of the choice of the coordinate frame. The chiral component of the texture can be separated from the achiral component by means of two fundamental invariants: Rogers's mean geodesic torsion [10,11],

$$
\rho=\mathbf{m} \cdot(\nabla \times \mathbf{m})
$$

and streamline curvature [11],

$$
k=|\mathbf{m} \times(\nabla \times \mathbf{m})| .
$$

\footnotetext{
*prybakov@kth.se
}

Published by the American Physical Society under the terms of the Creative Commons Attribution 4.0 International license. Further distribution of this work must maintain attribution to the author(s) and the published article's title, journal citation, and DOI. Funded by Bibsam.
For the cycloid in Fig. 1(a), $\rho(\mathbf{r})=0$ and therefore each part of this texture is achiral and can be superposed on its mirror image, while for the helix and its reflection shown in Fig. 1(b), the torsions are opposite in sign, $\rho(\mathbf{r})= \pm 2 \pi / L$, where $L$ is the period. Thus, the above helices have distinct chiralities.

The emergence of modulated ferromagnetic ordering may appear due to competing symmetric interactions between atoms in a lattice $[12,13]$. The multispin exchange interactions, in particular four-spin interactions, also provide a possible mechanism for the modulated texture stabilization beyond a pairwise scenario [14]. Most naturally, multispin exchange interactions can emerge in the form of two-site biquadratic or four-site spin interactions $[15,16]$. The delicate interplay between competing pairs and/or accounting for multispin interactions, leading to the stabilization of different magnetic textures, has been investigated both theoretically and experimentally in Refs. [17-22]. By virtue of symmetry of the above-mentioned and other symmetric interactions, the reflection of modulated magnetic texture is defined by degenerate energy states with opposite chirality, where a certain chirality is a consequence of spontaneous symmetry breaking.

An alternative mechanism of nucleation and stability of modulated ferromagnetic textures may be attributed to the presence of the pairwise Dzyaloshinskii-Moriya interaction (DMI), which is responsible for an asymmetric spin exchange, often discussed in magnetic systems where inversion symmetry is absent. Along with the magnetic ultrathin films and multilayers where the inversion symmetry is broken by natural means [5], the effect of DMI is pronounced in cubic crystals with chiral point group symmetry $T$, such as B20-type FeGe and $\mathrm{MnSi}$, in which a certain chirality of a magnetic helix is dictated by one of two possible enantiomorphic forms of the compound [23]. In this case, the corresponding magnetic Hamiltonian includes the Lifshitz invariants [2,24], and the chirality depends on the sign of their common factor. 


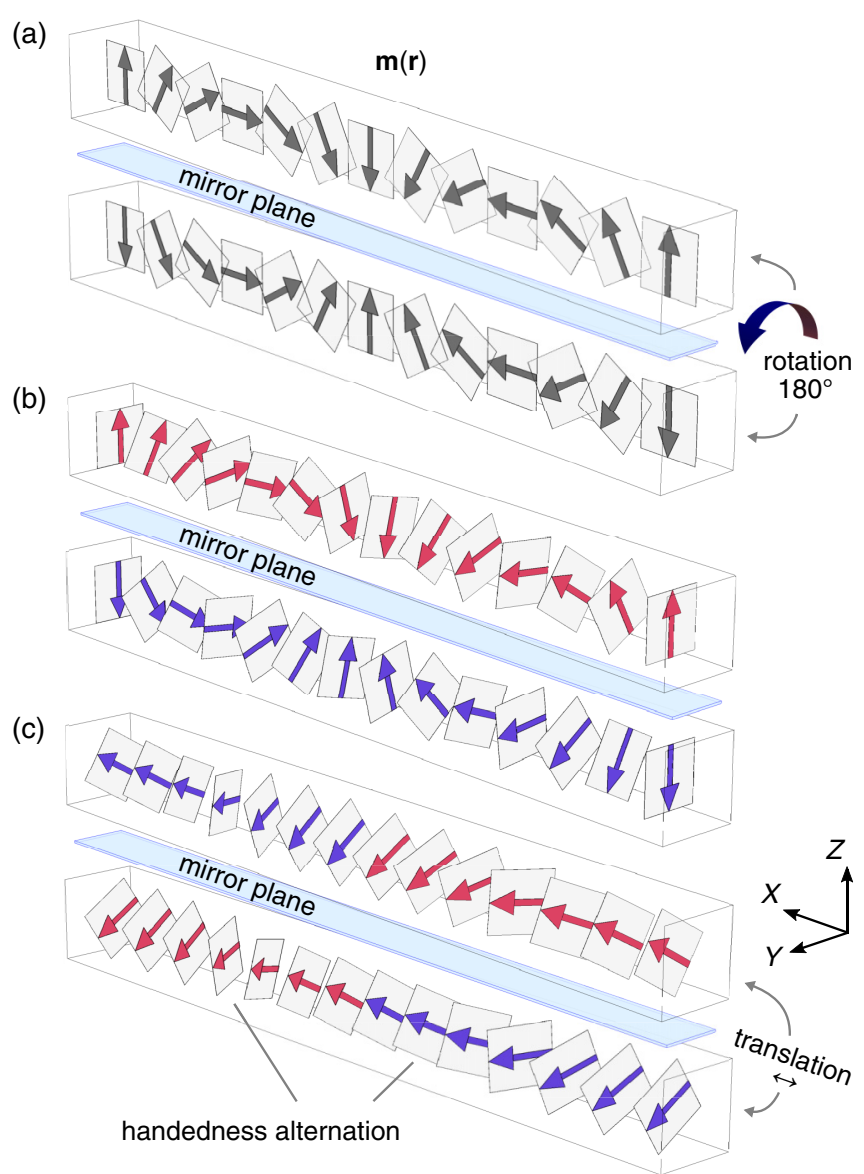

FIG. 1. Examples of periodic magnetic textures and their geometric reflections. (a) Cycloid, also known as Néel-type walls. (b) Helix, also known as Bloch-type walls. (c) Antichiral modulation found in the present work.

In this Letter, we predict a ferromagnetic ordering that is fundamentally different from those discussed above which we refer to as antichiral ferromagnetism. This term aims to reflect (i) that the chiral component $\rho(\mathbf{r})$ is significant, while the handedness alternates giving $\langle\rho(\mathbf{r})\rangle=0$, and (ii) this modulation is spontaneous and no explicit symmetry breaking is required for it.

Our analysis reveals that this magnetic ordering [see Fig. 1(c)] naturally appears in a bulk ferromagnet with the point group symmetry $T_{\mathrm{d}}$ owing to achiral crystal symmetry. This is a class of crystals in which many minerals are formed naturally $[25,26]$.

Note that in systems with a ferromagnetic ordering different from the antichiral one, the spatial alternation of handedness can be induced by an explicit symmetry breaking (such as a magnetic field, impurities, etc.). The corresponding magnetic textures are known as "fan," transverse conical, and others $[8,19,27-30]$. It is worth mentioning that the magnetic systems may exhibit a spatial alternation of chirality-related invariants other than $\rho$. For example, the so-called scalar spin chirality, where the relevant continuum invariant is the normal component of the vector of curvature [11], can alternate in two-dimensional systems [30].
The macroscopic robustness of a magnetic configuration is purely determined by its stability with respect to perturbations that violate spatial uniformity. If, for instance, inversion symmetry is broken, the spin alignment might gain a certain chirality due to spin-orbit driven antisymmetric DMI that contributes to the total energy with the terms linear with respect to the first spatial derivatives of magnetization. In general, the derivative linear contribution to the free energy of a ferromagnet can be cast in the form

$$
\mathcal{H}_{\nabla}=\int d \mathbf{r} \sum_{\alpha \beta} \Omega_{\alpha \beta}(\mathbf{m}) \nabla_{\alpha} m_{\beta},
$$

with the tensor $\Omega_{\alpha \beta}(-\mathbf{m})=-\Omega_{\alpha \beta}(\mathbf{m})$ being odd under magnetization inversion [31], while $\alpha, \beta$ correspond to the spatial indices. By expanding $\Omega_{\alpha \beta}(\mathbf{m})=\sum_{\gamma} \Omega_{\alpha \beta \gamma}^{(1)} m_{\gamma}+$ $\sum_{\gamma \delta \varepsilon} \Omega_{\alpha \beta \gamma \delta \varepsilon}^{(3)} m_{\gamma} m_{\delta} m_{\varepsilon}$ in powers of $\mathbf{m}$ and restricting to linear and cubic contributions only, one arrives at

$$
\begin{aligned}
\mathcal{H}_{\nabla}= & \frac{1}{2} \int d \mathbf{r} \sum_{\alpha \beta \gamma}\left[\mathcal{D}_{\alpha \beta \gamma}^{S} \nabla_{\alpha}\left(m_{\beta} m_{\gamma}\right)+\mathcal{D}_{\alpha \beta \gamma}^{A} \mathcal{L}_{\gamma \beta}^{(\alpha)}\right] \\
& +\int d \mathbf{r} \sum_{\alpha \beta \gamma \delta \varepsilon} \Omega_{\alpha \beta \gamma \delta \varepsilon}^{(3)} m_{\gamma} m_{\delta} m_{\varepsilon} \nabla_{\alpha} m_{\beta},
\end{aligned}
$$

where for convenience we have separated the terms arising due to $\Omega^{(1)}$ into two parts, namely symmetric terms, $\nabla_{\alpha}\left(m_{\beta} m_{\gamma}\right)$, and terms given in the form of Lifshitz invariants, $\mathcal{L}_{\beta \gamma}^{(\alpha)}=m_{\beta} \nabla_{\alpha} m_{\gamma}-m_{\gamma} \nabla_{\alpha} m_{\beta}$ [32]. Note that as the symmetric contribution can be expressed in terms of surface integrals via Stokes' theorem [31], its impact on the magnetic ordering in bulk crystals can be discarded.

To proceed, we analyze (4) based on symmetry grounds (see Appendix A). The solution for the $T_{\mathrm{d}}$ point group symmetry is trivial with respect to Lifshitz invariants, $\mathcal{D}_{\alpha \beta \gamma}^{A}=0$, whereas in the case of $\Omega^{(3)}$ one can identify four independent components with the corresponding invariants given by

$$
\begin{aligned}
& \sum, \nabla_{\alpha}\left(m_{\beta}^{3} m_{\gamma}+m_{\beta} m_{\gamma}^{3}\right), \quad \sum \nabla_{\alpha}\left(m_{\alpha}^{2} m_{\beta} m_{\gamma}\right), \\
& \sum_{\alpha}, m_{\alpha} m_{\beta} \nabla_{\gamma}\left(\mathbf{m}^{2}\right), \\
& m_{x} m_{y} m_{z} \nabla \cdot \mathbf{m},
\end{aligned}
$$

where $\sum$, denote the sum over $(\alpha, \beta, \gamma) \in$ $\{(x, y, z),(y, z, x),(z, x, y)\}$. The invariant (5c) was first reported by Ado et al. [33,34], whereas we derive the complete set $(5 a)-(5 c)$. After integrating by parts both terms (5a) can be discarded for the same reasons as the terms $\nabla_{\alpha}\left(m_{\beta} m_{\gamma}\right)$ in the above analysis. Note that within the micromagnetic approach magnetization is described in terms of a unit vector $|\mathbf{m}(\mathbf{r})|=1$ and thus the invariant (5b) vanishes as well. Therefore we end up with (5c) as the only relevant term for Eq. (3).

We proceed with a microscopic analysis of the term $(5 \mathrm{c})$. Due to anisotropy of the crystal, shown in Fig. 2(a), the microscopic Hamiltonian for classical spins $\mathbf{s}_{i}$ must be equipped with direction vectors. The possible term that corresponds to such a contribution to the micromagnetic energy may be cast 
(a)

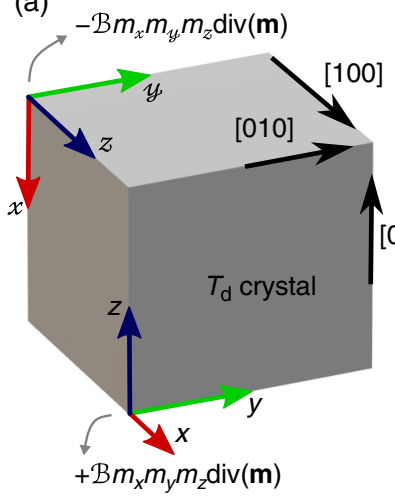

(b)

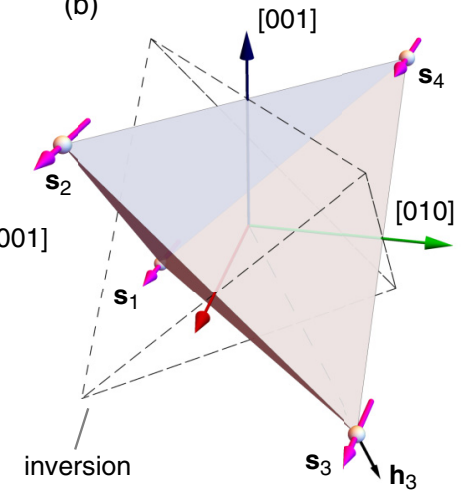

FIG. 2. (a) Sketch of bulk crystal with achiral point group symmetry $T_{\mathrm{d}}$. By virtue of rotoreflection $S_{4}$ invariants, the sign of the phenomenological constant $\mathcal{B}$ alternates at $90^{\circ}$ rotations of the coordinate frame. (b) Possible structural building block, satisfying full tetrahedral symmetry. The dashed line denotes the block arising from the inversion, thereby emphasizing the lack of the inversion symmetry.

in the form

$$
H=\beta\left(\sum_{i=1}^{4} \mathbf{s}_{i} \cdot \mathbf{h}_{i}\right) \sum_{i=1}^{4}\left(\mathbf{s}_{i} \cdot \mathbf{h}_{i}\right)^{3},
$$

where unit vectors $\mathbf{h}_{i}$ are aligned with the bonds connecting the center of a tetrahedron and the corresponding vertex [see Fig. 2(b)], while $\beta$ represents the strength of the interaction. Note that multispin interactions whose Hamiltonian contains direction vectors were recently discovered in B20-type cubic chiral magnets [35]. Moreover, we expect that a certain insight on the nature of pairwise and multispin interactions [36,37] can be gained on the basis of first-principles calculations that could clarify the microscopic origin of the term $(5 c)$.

We base our subsequent analysis on the minimal model of a tetrahedral ferromagnet that contains an exchange interaction and Ado interaction (5c),

$$
\mathcal{H}=\int d \mathbf{r}\left(A|\nabla \mathbf{m}|^{2}+\mathcal{B} m_{x} m_{y} m_{z} \nabla \cdot \mathbf{m}\right),
$$

where $A$ is the exchange stiffness. Additionally, where it is specifically stated, we will take into account symmetryallowed cubic anisotropy with energy density in the form $K\left(m_{x}^{2} m_{y}^{2}+m_{x}^{2} m_{z}^{2}+m_{y}^{2} m_{z}^{2}\right)$. Without loss of generality, we assume $\mathcal{B}>0$, since the sign of this constant depends on the choice of the coordinates (see Fig. 2). To identify the lowest-energy state, as well as stable excited configurations, we perform a numerical minimization of the energy (see Appendix B for details).

In Figs. 3(b)-3(d) we provide relevant energy minimum configurations that emerge from an initial guess with randomly oriented spins, as depicted in Fig. 3(a). Interestingly, the magnetic structure in Fig. 3(d) was found to be the energetically most favorable. Reducing the spatial dimension to two, while increasing the size of the modeling area, we also obtained that the type of modulations depicted in Fig. 3(d) is the most energetically favorable configuration. Note that the presence of cubic anisotropy leads to similar results, but noticeably affects the optimal period of the observed modulation [see Fig. 3(e)]. A closer inspection of the found magnetic (a)

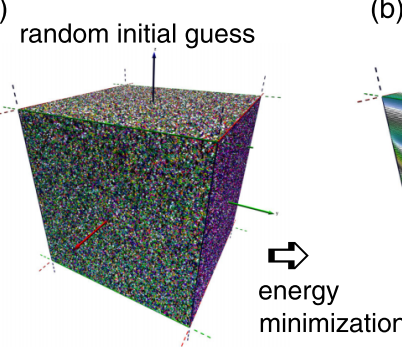

(e)

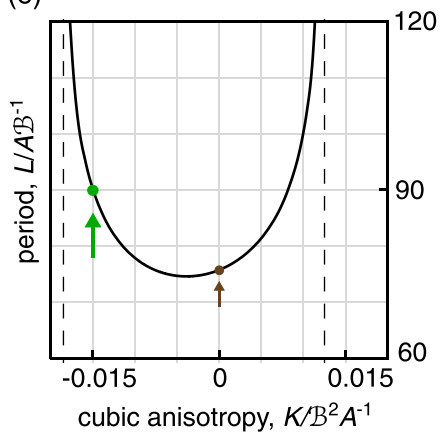

(b)

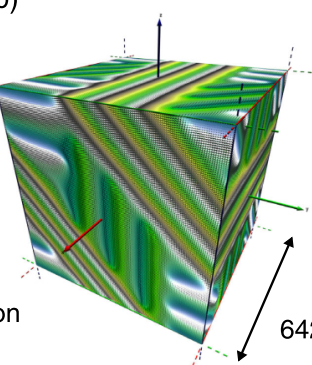

(f)

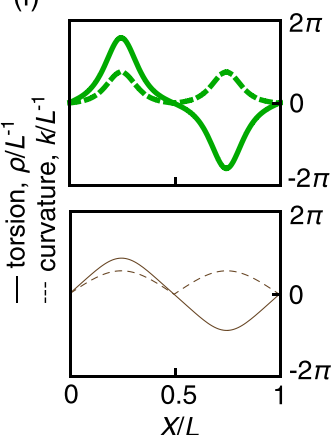

(c)

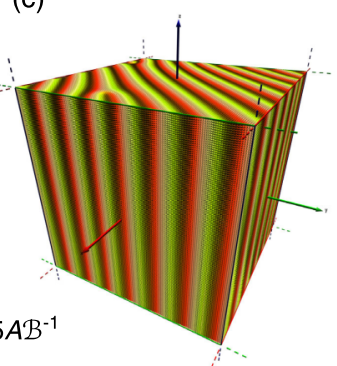

(g)

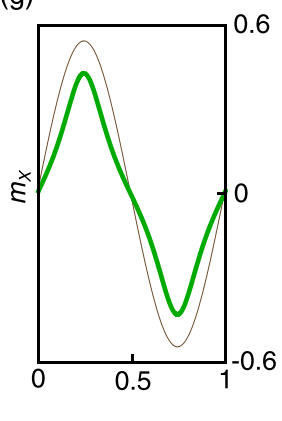

(d)

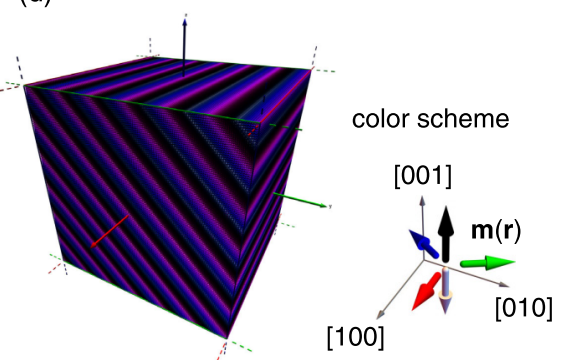

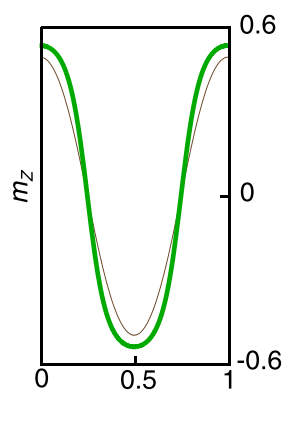

FIG. 3. (a) Initial guess with chaotically oriented magnetization for the cube under periodic boundary conditions. (b)-(d) Typical numerical solutions corresponding to an energy minimum: multidomain structure, modulations with edge dislocations, and perfect modulations, respectively. (e) Dependence of the optimal period of the antichiral modulation on cubic anisotropy. (f) Torsion and curvature values, and (g) magnetization projections plotted at each point along the modulation direction. For (f) and (g) the thicker line (green) corresponds to the case of nonzero cubic anisotropy $K=-0.015 \mathcal{B}^{2} / A$, for which a three-dimensional view is presented in Fig. 1(c). 


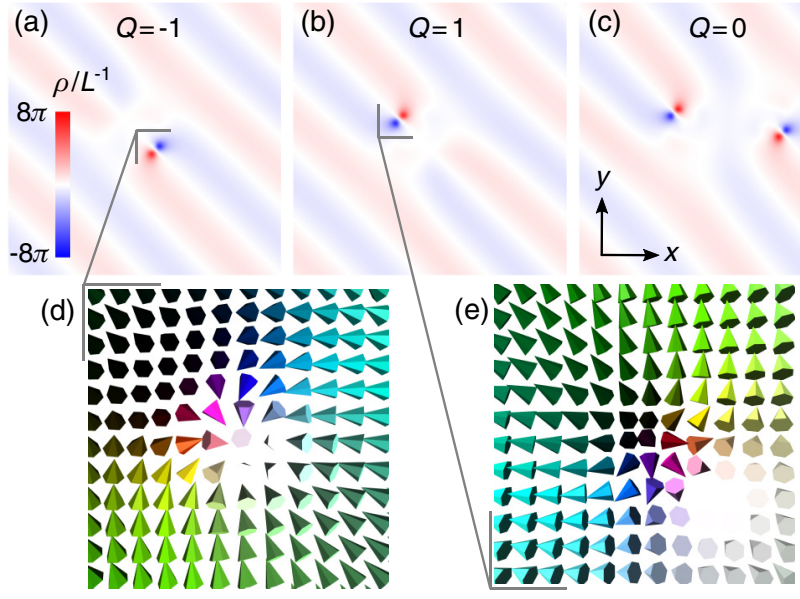

FIG. 4. (a)-(c) The torsion $\rho(x, y)$ calculated for found excitations: skyrmions having negative and positive topological charge, and clusters composed by their pairs, respectively. The approximate energy values of these states are 12.02, 12.02, and 23.58, respectively. The energy is calculated relative to the global minimum in units of $2 A t$, where $t$ is the thickness in the $z$ direction. (d), (e) Magnetization near the marked central region in (a) and (b), respectively.

ordering is visualized in Figs. 1(c) and 3(g). It can be clearly seen that locally in space the magnetic spiral has distinct chirality. The sign of the calculated torsion $\rho$ varies in space [Fig. 3(f)], resulting in a bichiral configuration that vanishes when averaged over a modulation period. On this account we label this state with alternating local chiralities as antichiral. For data points and an analytical approximation of the ground state, see Ref. [38] and Appendix C, respectively. The spatial orientation of the texture is sixfold degenerate, since the modulation axis $X$ can be parallel to any vector from the $\langle 110\rangle$ family. The polarity of modulation is twofold degenerate, meaning that if, for example, $X$ is parallel to [110], then the vector $\langle\mathbf{m}(\mathbf{r})\rangle$ is directed along either [110] or [110]. The resulting 12 -fold degeneracy should be spontaneously broken in this model. In a finite sample, we expect that the significant net magnetization and stray fields will favor the split into the domains.

Having established the ground state we proceed with the discussion on the possibility of stable excited configurations, apart from those found by numerical energy minimization from the random state. Notably, several classes of magnets allow particlelike topological excitations, such as magnetic skyrmions $[5,7,32]$. In order to resolve numerically whether the present system has similar type excitations we use the vortexlike ansatz [32] as an initial guess, superimposing modulation mimicking the ground state discussed above. This approach is justified since there are systems where skyrmions are embedded in the modulated state [39-41]. Our findings suggest that the model (7) possesses stable localized states with an integer topological charge $Q=(4 \pi)^{-1} \int d x d y \mathbf{m} \cdot\left[\partial_{x} \mathbf{m} \times \partial_{y} \mathbf{m}\right]$ (see Fig. 4). Interestingly, contrasting to their counterparts in chiral magnets, skyrmions that we obtain in this model do not have a predefined chirality, but instead, they have two spots with strong left-handed and right-handed torsion.
We demonstrated that the magnetic ordering in crystals with the $T_{\mathrm{d}}$ (tetrahedral class) point group can be of a different type, that we refer to as antichiral ferromagnetism. As discussed in the Supplemental Material, there are many compounds of this class containing magnetic elements $(\mathrm{Cr}, \mathrm{Mn}$, $\mathrm{Fe}, \mathrm{Co}$, and Ni) [38]. Among the methods that may be used to probe the predicted antichiral ordering, one can highlight small-angle neutron scattering, spin-polarized scanning probe microscopy [42], as well as the electron magnetic circular dichroism technique [43].

Our numerical findings reveal that the antichiral ground state has a set of stable topologically nontrivial excitations in the form of edge dislocations and skyrmions. These excitations are antichiral in contrast to their chiral counterparts in systems with a Dzyaloshinskii-Moriya interaction. Their properties pose an intriguing question that open up interesting theoretical and experimental research directions.

Note added. Ado et al. report an independent study on model (7) [44].

F.N.R. would like to thank A. B. Borisov for useful discussions. A.P. acknowledges the support from Russian Foundation for Basic Research Project No. 19-32-60020 and the Russian Federation President Scholarship for Young Scientists (SP-1640.2021.5). E.B. and F.N.R. were supported by the Swedish Research Council Grants No. 642-2013-7837, No. 2016-06122, No. 2018-03659, and Göran Gustafsson Foundation for Research in Natural Sciences. O.E. acknowledges support from the Swedish Research Council, the Knut and Alice Wallenberg foundation, the Foundation for Strategic Research, the European Research Council, and eSSENCE. O.E. also acknowledges help by Dr. A. Vishina for providing the list of materials with $T_{\mathrm{d}}$ symmetry, discussed in the Supplemental Material.

\section{APPENDIX A: SYMMETRY ANALYSIS METHOD}

Given a point group symmetry with generators $\mathcal{R}^{(\mu)}$, a tensorial structure is dictated by the symmetry relations

$$
\Omega_{\alpha_{1} \alpha_{2} \cdots \alpha_{n}}^{(n)}=\mathcal{R}_{\alpha_{1} \beta_{1}}^{(\mu)} \mathcal{R}_{\alpha_{2} \beta_{2}}^{(\mu)} \cdots \mathcal{R}_{\alpha_{n} \beta_{n}}^{(\mu)} \Omega_{\beta_{1} \beta_{2} \cdots \beta_{n}}^{(n)},
$$

where $\mathcal{R}_{\alpha \beta}^{(\mu)}$ are orthogonal matrices of three-dimensional irreducible representations for each element $\mu$ of the group. The corresponding matrices for the tetrahedral point group $T_{\mathrm{d}}$ can be found, e.g., in Refs. [45-47]. Reduction of the linear system (A1) identifies zero and nonzero components of the tensor $\Omega$.

\section{APPENDIX B: NUMERICAL ENERGY MINIMIZATION}

The continuous model as yielded by Eq. (7) was discretized using a rectangular grid under periodic boundary conditions. We implemented a discretization scheme giving an eighth order of accuracy by generalizing the approach of Donahue and McMichael [48]. The typical number of grid points in each dimension ranged from 140 to 160 . For minimization we used a GPU-parallelized nonlinear conjugate gradient algorithm [49], while the constraint $|\mathbf{m}|=1$ was satisfied by means of the special use of stereographic projections (see, e.g., Supplemental Material in Ref. [50]). 


\section{APPENDIX C: ANALYTICAL APPROXIMATION OF THE GROUND STATE}

Cycloid [Fig. 1(a)], helix [Fig. 1(b)], screws, and cones $[8,19,29,41]$ are analytically described by the Rodrigues' rotation formula with an $\mathbf{r}$-dependent angle,

$$
\begin{aligned}
\mathbf{m}(\mathbf{r})= & \mathbf{v} \cos \theta(\mathbf{r})+(\mathbf{p} \times \mathbf{v}) \sin \theta(\mathbf{r}) \\
& +\mathbf{p}(\mathbf{p} \cdot \mathbf{v})[1-\cos \theta(\mathbf{r})],
\end{aligned}
$$

where $\mathbf{p}$ and $\mathbf{v}$ are some unit vectors. Despite its versatility, expression $(\mathrm{C} 1)$ is rather unsuitable for approximating the antichiral states found here. The following approximation is noticeably more accurate,

$$
\begin{aligned}
& m_{X} \approx a \sin (2 \pi X / L), \quad m_{Z} \approx b \cos (2 \pi X / L), \\
& m_{Y}=\sqrt{1-m_{X}^{2}-m_{Z}^{2}},
\end{aligned}
$$

where the parameters $a$ and $b$ are subject to optimization (for example, for $K=0, a \approx 0.54, b \approx 0.5$ ), and period $L$ can be taken from Fig. 3(e). For XYZ coordinates, see Fig. 1(c). It is important to note that the elliptic conical approximation (C2), although better than $(\mathrm{C} 1)$, deviates from the true texture in a noticeable manner for some $K$ values.
[1] D. E. Kharzeev, The chiral magnetic effect and anomalyinduced transport, Prog. Part. Nucl. Phys. 75, 133 (2014).

[2] P. Bak and M. H. Jensen, Theory of helical magnetic structures and phase transitions in MnSi and FeGe, J. Phys. C 13, L881 (1980).

[3] Y. Tokunaga, X. Z. Yu, J. S. White, H. M. Rønnow, D. Morikawa, Y. Taguchi, and Y. Tokura, A new class of chiral materials hosting magnetic skyrmions beyond room temperature, Nat. Commun. 6, 7638 (2015).

[4] S. S. P. Parkin, M. Hayashi, and L. Thomas, Magnetic domainwall racetrack memory, Science 320, 190 (2008).

[5] A. Fert, V. Cros, and J. Sampaio, Skyrmions on the track, Nat. Nanotechnol. 8, 152 (2013).

[6] K. Koumpouras, A. Bergman, O. Eriksson, and D. Yudin, A spin dynamics approach to solitonics, Sci. Rep. 6, 25685 (2016).

[7] C. Back, V. Cros, H. Ebert, K. Everschor-Sitte, A. Fert, M. Garst, T. Ma, S. Mankovsky, T. L. Monchesky, M. Mostovoy, N. Nagaosa, S. S. P. Parkin, C. Pfleiderer, N. Reyren, A. Rosch, Y. Taguchi, Y. Tokura, K. von Bergmann, and J. Zang, The 2020 skyrmionics roadmap, J. Phys. D 53, 363001 (2020).

[8] Y. Kitano and T. Nagamiya, Magnetization process of a screw spin system. II, Prog. Theor. Phys. 31, 1 (1964).

[9] Y. A. Izyumov, Modulated, or long-periodic, magnetic structures of crystals, Sov. Phys. Usp. 27, 845 (1984).

[10] R. A. P. Rogers, Some differential properties of the orthogonal trajectories of a congruence of curves, with an application to curl and divergence of vectors, Proc. R. Ir. Acad., Sect. A: Math. Phys. Sci. 29, 92 (1912).

[11] Y. Aminov, The Geometry of Vector Fields (Gordon and Breach, Amsterdam, 2000).

[12] J. Villain, La structure des substances magnetiques, J. Phys. Chem. Solids 11, 303 (1959).

[13] A. Yoshimori, A new type of antiferromagnetic structure in the rutile type crystal, J. Phys. Soc. Jpn. 14, 807 (1959).

[14] S. Paul, S. Haldar, S. von Malottki, and S. Heinze, Role of higher-order exchange interactions for skyrmion stability, Nat. Commun. 11, 4756 (2020).

[15] M. Takahashi, Half-filled Hubbard model at low temperature, J. Phys. C: Solid State Phys. 10, 1289 (1977).

[16] A. H. MacDonald, S. M. Girvin, and D. Yoshioka, $\frac{t}{U}$ expansion for the Hubbard model, Phys. Rev. B 37, 9753 (1988).

[17] Ph. Kurz, G. Bihlmayer, K. Hirai, and S. Blügel, Three-Dimensional Spin Structure on a Two-Dimensional
Lattice: $\mathrm{Mn} / \mathrm{Cu}(111)$, Phys. Rev. Lett. 86, 1106 (2001).

[18] S. Heinze, K. von Bergmann, M. Menzel, J. Brede, A. Kubetzka, R. Wiesendanger, G. Bihlmayer, and S. Blügel, Spontaneous atomic-scale magnetic skyrmion lattice in two dimensions, Nat. Phys. 7, 713 (2011).

[19] A. O. Leonov and M. Mostovoy, Multiply periodic states and isolated skyrmions in an anisotropic frustrated magnet, Nat. Commun. 6, 8275 (2015).

[20] S. Hayami, R. Ozawa, and Y. Motome, Effective bilinearbiquadratic model for noncoplanar ordering in itinerant magnets, Phys. Rev. B 95, 224424 (2017).

[21] N. Romming, H. Pralow, A. Kubetzka, M. Hoffmann, S. von Malottki, S. Meyer, B. Dupé, R. Wiesendanger, K. von Bergmann, and S. Heinze, Competition of DzyaloshinskiiMoriya and Higher-Order Exchange Interactions in $\mathrm{Rh} / \mathrm{Fe}$ Atomic Bilayers on $\operatorname{Ir}(111)$, Phys. Rev. Lett. 120, 207201 (2018).

[22] S. Meyer, M. Perini, S. von Malottki, A. Kubetzka, R. Wiesendanger, K. von Bergmann, and S. Heinze, Isolated zero field sub-10 nm skyrmions in ultrathin Co films, Nat. Commun. 10, 3823 (2019).

[23] S. M. Stishov and A. E. Petrova, Itinerant helimagnet MnSi, Phys. Usp. 54, 1117 (2011).

[24] V. G. Bar'yakhtar and E. P. Stefanovsky, Spin wave spectrum in antiferromagnets having a spiral magnetic structure, Sov. Phys. Solid State 11, 1566 (1970).

[25] S. Sportouch and M. G. Kanatzidis, $\mathrm{Th}_{3} \mathrm{Co}_{3} \mathrm{Sb}_{4}$ : A new room temperature magnet, J. Solid State Chem. 162, 158 (2001).

[26] S. Lee, H. Xu, H. Xu, R. Jacobs, and D. Morgan, Valleyite: A new magnetic mineral with the sodalite-type structure, Am. Mineral. 104, 1238 (2019).

[27] T. Komatsubara, T. Suzuki, and E. Hirahara, Magnetization process and spin-structure diagram in manganese phosphide single crystal, J. Phys. Soc. Jpn. 28, 317 (1970).

[28] K. Siratori, J. Akimitsu, E. Kita, and M. Nishi, A method of controlling the sense of the screw spin structure, J. Phys. Soc. Jpn. 48, 1111 (1980).

[29] S.-Z. Lin and S. Hayami, Ginzburg-Landau theory for skyrmions in inversion-symmetric magnets with competing interactions, Phys. Rev. B 93, 064430 (2016).

[30] S. Hayami, S.-Z. Lin, Y. Kamiya, and C. D. Batista, Vortices, skyrmions, and chirality waves in frustrated Mott insulators 
with a quenched periodic array of impurities, Phys. Rev. B 94, 174420 (2016).

[31] I. A. Ado, A. Qaiumzadeh, A. Brataas, and M. Titov, Chiral ferromagnetism beyond Lifshitz invariants, Phys. Rev. B 101, 161403(R) (2020).

[32] A. N. Bogdanov and D. A. Yablonskii, Thermodynamically stable "vortices" in magnetically ordered crystals. the mixed state of magnets, Sov. Phys. JETP 68, 101 (1989).

[33] I. Ado, presentation delivered at the SPICE-SPIN+X Seminars, Mainz, Germany, February 2020 (unpublished).

[34] M. Titov, online presentation delivered at the Ultrafast Dynamics of Correlated Electrons in Solids workshop, May 2020, https://youtu.be/OgpP13ZcDRw.

[35] S. Grytsiuk, J.-P. Hanke, M. Hoffmann, J. Bouaziz, O. Gomonay, G. Bihlmayer, S. Lounis, Y. Mokrousov, and S. Blügel, Topological-chiral magnetic interactions driven by emergent orbital magnetism, Nat. Commun. 11, 511 (2020).

[36] R. Cardias, A. Szilva, M. M. Bezerra-Neto, M. S. Ribeiro, A. Bergman, Y. O. Kvashnin, J. Fransson, A. B. Klautau, O. Eriksson, and L. Nordström, First-principles DzyaloshinskiiMoriya interaction in a non-collinear framework, Sci. Rep. 10, 20339 (2020).

[37] S. Mankovsky, S. Polesya, and H. Ebert, Extension of the standard Heisenberg Hamiltonian to multispin exchange interactions, Phys. Rev. B 101, 174401 (2020).

[38] See Supplemental Material at http://link.aps.org/supplemental/ 10.1103/PhysRevB.104.L020406 for data points and examples of compounds with the $T_{\mathrm{d}}$ crystallographic point group.

[39] J. Müller, J. Rajeswari, P. Huang, Y. Murooka, H. M. Rønnow, F. Carbone, and A. Rosch, Magnetic Skyrmions and Skyrmion Clusters in the Helical Phase of $\mathrm{Cu}_{2} \mathrm{OSeO}_{3}$, Phys. Rev. Lett. 119, 137201 (2017).
[40] P. Schoenherr, J. Müller, L. Köhler, A. Rosch, N. Kanazawa, Y. Tokura, M. Garst, and D. Meier, Topological domain walls in helimagnets, Nat. Phys. 14, 465 (2018).

[41] M. Speight and T. Winyard, Skyrmions and spin waves in frustrated ferromagnets at low applied magnetic field, Phys. Rev. B 101, 134420 (2020).

[42] M. F. Crommie, C. P. Lutz, and D. M. Eigler, Imaging standing waves in a two-dimensional electron gas, Nature (London) $\mathbf{3 6 3}$, 524 (1993).

[43] P. Schattschneider, S. Rubino, C. Hébert, J. Rusz, J. Kuneš, P. Novák, E. Carlino, M. Fabrizioli, G. Panaccione, and G. Rossi, Detection of magnetic circular dichroism using a transmission electron microscope, Nature (London) 441, 486 (2006).

[44] I. A. Ado, O. Tchernyshyov, and M. Titov, Non-collinear ground state from a four-spin chiral exchange in a tetrahedral magnet, arXiv:2012.07666.

[45] P. Atkins and R. Friedman, Molecular Quantum Mechanics (Oxford University Press, Oxford, U.K., 2005).

[46] Y. Xu and X. Chen, Exploring the group representation theory of the full symmetry of regular tetrahedron, arXiv:1910.07143 (2019).

[47] D. Arovas, Lecture Notes on Group Theory in Physics (University of California, San Diego, 2020), https://courses.physics.ucsd.edu/2016/Spring/physics220/ LECTURES/GROUP_THEORY.pdf.

[48] M. Donahue and R. McMichael, Exchange energy representations in computational micromagnetics, Phys. B: Condens. Matter 233, 272 (1997).

[49] F. N. Rybakov and E. Babaev, Excalibur software, http:// quantumandclassical.com/excalibur/.

[50] F. N. Rybakov, A. B. Borisov, S. Blügel, and N. S. Kiselev, New Type of Stable Particlelike States in Chiral Magnets, Phys. Rev. Lett. 115, 117201 (2015). 